\title{
Medication Related Osteonecrosis of Jaw
}

\author{
Prasanthi Sitaraman ${ }^{1}$, Arvind Muthukrishnan ${ }^{2}$ \\ ${ }^{1}$ Department of Oral Medicine and Radiology, Saveetha Dental College, Tamilnadu, India. ${ }^{2}$ Department of Oral \\ Medicine and Radiology, Saveetha Dental College, Chennai, Tamilnadu, India.
}

\section{PRESENTATION OF CASE}

A 41 year old female patient reported with the complaint of pain in the right posterior mandibular region for the past 5 months. History revealed that patient was diagnosed with breast cancer before 4 years following which she underwent mastectomy, radiotherapy and chemotherapy. PET / CT taken post chemotherapy showed bone metastasis to the lumbar spine for which she was prescribed I.V. zoledronic acid to be administered every 3 months. Patient was under I.V. zoledronic acid for a total of 36 months.

Patient underwent surgery for extraction of impacted lower third molar before 7 months. The extraction site did not completely heal following which the tooth adjacent to the extraction site became mobile. The mobile tooth was also extracted before 5 months following which she developed pain and swelling in the region. Patient was diagnosed with osteoradionecrosis and underwent curettage and antiseptic dressing for 2 months. However, there was no reduction in pain. Pain was gradual in onset, continuous, gnawing, in the extraction site, aggravated by mastication and relieved by medication.

On clinical examination, non - healing extraction socket was evident in 47,48 region. A single, oval ulcer measuring about $0.5 \mathrm{~cm} * 0.5 \mathrm{~cm}$ was evident in the medial surface of the retro molar area in 48 region. The margins of the ulcer was well defined and the floor was covered with granulation tissue with erythematous surrounding mucosa. No discharge is evident from the ulcer. On palpation, the ulcer is tender. The floor of the ulcer revealed necrotic bone (Figure 1).

The patient was subsequently subjected to Orthopantomogram (OPG) and Computed tomography (CT) (Figure 2). OPG shows missing 47, 48 and loss of alveolar bone and extending from the distal surface of 46 and posteriorly to the retromolar region, inferiorly to about $2 \mathrm{~mm}$ from the mandibular canal. The affected bone has irregular margins and comparatively radiolucent to the surrounding bone on the posterior margin. The CT - axial, coronal, sagittal sections with 3D reconstruction showed post-operative bone defect in the lower right second and third molar region from the previous curettage.

In consultation with the physician, intravenous bisphosphonate was discontinued. Antibiotics clindamycin (300 mg, B.I.D.) was coupled with pentoxiphylline (400 mg, O.D.) with supplementary povidone iodine and Chlorhexidine gluconate mouth gargle was prescribed. The exposed bone was subsequently surgically removed after 15 days of medical management (Figure 3).

Based on the chief complaint, medical history and radiographic findings, a diagnosis of stage 2 Medication Related Osteonecrosis of Jaw (MRONJ) was given. The patient is under regular follow-up.

Anti-resorptive drugs are those drugs that reduce bone loss by decreasing osteoplastic bone resorption. They are used in the treatment of various medical conditions, including osteoporosis, multiple myeloma, and breast cancer with skeletal metastasis, because they reduce bone pain, hyperkalaemia, and the risk of pathologic fractures. There are 5 classes of antiresorptive drugs, of which bisphosphonates are increasingly associated with Osteonecrosis of jaw. This condition was named as Bisphosphonate related osteonecrosis of jaw (BRONJ), later renamed as Medication related osteonecrosis of jaw (MRONJ) following identification of non-bisphosphonate related aetiology such as antiangiogenic agents such as bevacizumab, aflibercept and sunitinib.
Corresponding Author: Dr. Prasanthi Sitaraman. Department of Oral Medicine and Radiology, Saveetha Dental College, Saveetha Institute of Medical and Technical Sciences (SIMATS), 162, Poonamallee High Road, Chennai - 600077, Tamilnadu, India. E-mail: prasha92@yahoo.com

DOI: $10.14260 / j e m d s / 2020 / 602$

How to Cite This Article:

Sitaraman P, Muthukrishnan A. Medication related osteonecrosis of jaw. J Evolution Med Dent Sci 2020;9(37):2770-2772, DOI: 10.14260/jemds/2020/602

Submission 13-06-2020,

Peer Review 07-08-2020,

Acceptance 13-08-2020,

Published 14-09-2020.

Copyright (C) 2020 Prasanthi Sitaraman et al. This is an open access article distributed under Creative Commons Attribution License [Attribution 4.0 International (CC BY 4.0)] 
This case report describes a 41-year-old female patient on zoledronic acid therapy following bone metastasis from breast cancer who developed osteonecrosis of jaw following extraction of right lower wisdom teeth. Careful history taking to review medication in oncology patients with history of chemotherapy (antiresorptive drugs) and liaison with medical oncologist prior to any invasive dental treatment on these patients with bisphosphonates / denosumab / antiangiogenic drugs is absolutely necessary to prevent the occurrence of consequences such as MRONJ. Role of oral medicine physicians in prevention and treating osteochemonecrosis is inevitable as early prevention and accurate treatment increases quality of life in these groups of patients.

Antiresorptive drugs are osteotropic agents used for their ability to prevent bone resorption in patients with osteoporosis, metabolic bone diseases and hypercalcemia. The classes of antiresorptive drugs are bisphosphonates, oestrogens, selective oestrogen receptor modulators, calcitonin, and monoclonal antibodies. There are three generations of bisphosphonates, most potent of these drugs are the second and the third generation - alendronate and zoledronate ${ }^{1}$. Other common indications for the use of BPNs is metastatic cancer of bone. First described by Marx in 2003, osteonecrosis of jaw is the most frequently reported adverse effect of $\mathrm{BPNs}^{2}$. The terms bisphosphonate - related osteonecrosis of the jaw (BRONJ) and antiresorptive agent related ONJ (ARONJ) has been advocated. The AAOMS position paper (2014) favours the use of the term medication-related osteonecrosis of the jaw (MRONJ) after identification of other drugs like anti - angiogenic drugs as the cause of osteonecrosis $^{3}$. MRONJ is considered a severe adverse drug reaction, consisting of progressive bone destruction in the maxillofacial region of patients. Patients under zoledronic acid and denosumab have shown to have increased risk of developing MRONJ than other antiresorptive drugs ${ }^{3}$. This case study illustrates a 41-year-old female patient under I.V. zoledronic acid who reported with jaw pain post extraction of wisdom tooth.
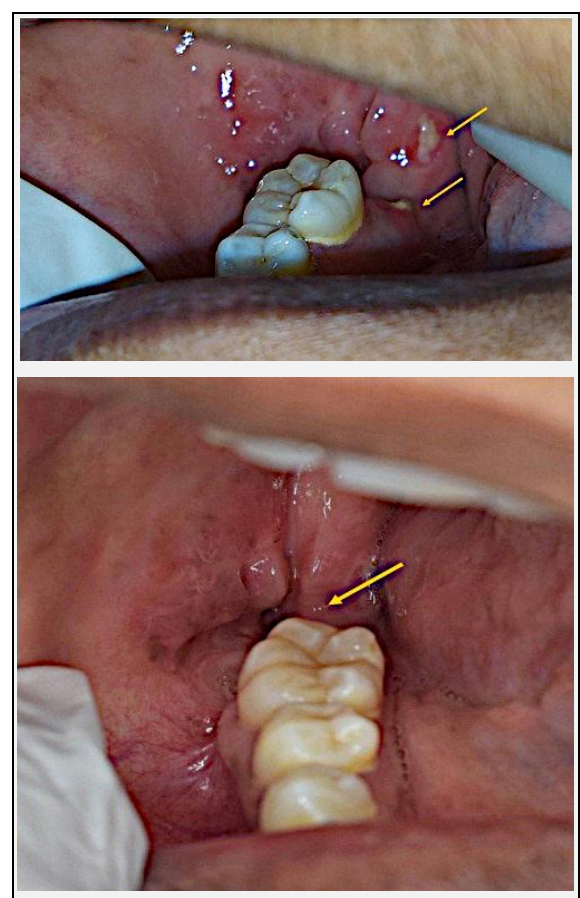

Figure 1.

Floor of the Ulcer Revealing Necrotic Bone
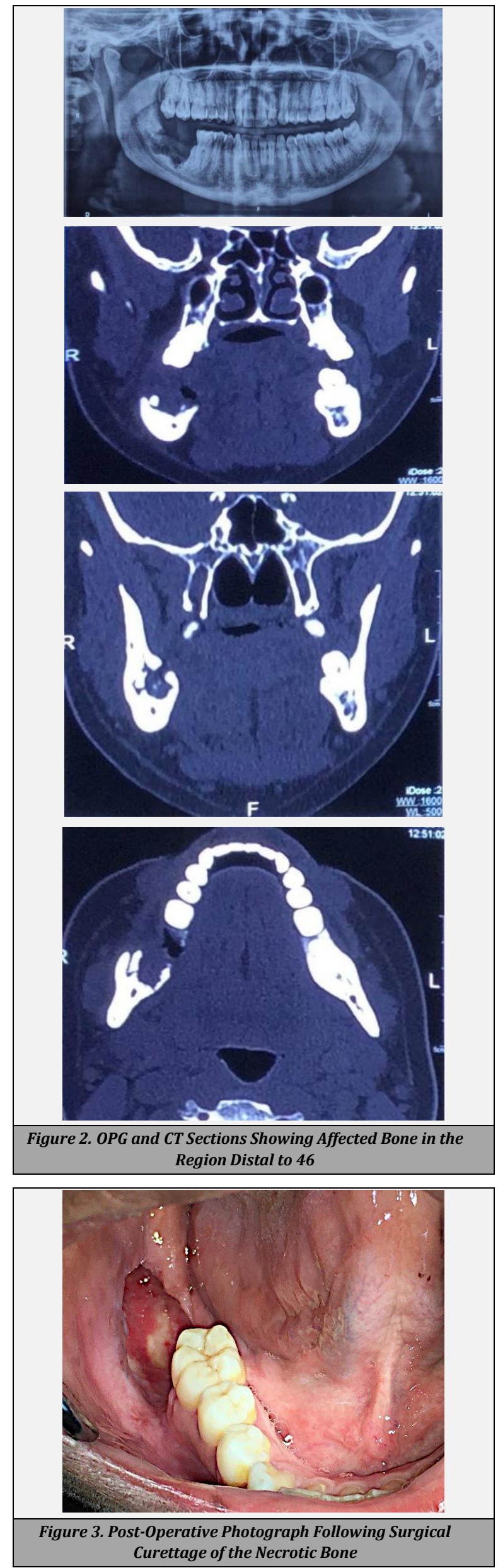


\section{DISCUSSION}

MRONJ is a consequence of use of antiresorptive drugs and antiangiogenic drugs for varied therapeutic indications. The pathophysiology is not well understood. However, multiple hypothesis has been put forward to elucidate the localization to the jaws and not the other bones of the body. These include altered remodelling, trauma, suppression of osteoclastic activity, inhibition of angiogenesis, decreased vascularity to the jaw bone and suppression of immunity due to previous treatments for treating cancer. ${ }^{4}$ Risk factors like the drug, mode of administration, length of therapy, age and gender of the patient, other co - morbidities, local factors like trauma from dentures, inflammatory disease have to be considered. Multiple studies have reported that in about $52-61 \%$ of cases, dental extraction acts as trigger for MRONJ which is similar to that of our case.5,6 Detailed history with the duration of treatment with antiresorptive and antiangiogenic medication has to be recorded prior to any dental procedure.

Communication with the oncologist / physician is unquestionably needed to prevent complications such as MRONJ.7

Case definition of BRONJ / ARONJ / MRONJ has not changed since the AAOMS position paper, 2009.3,8 Presence current or previous treatment with a bisphosphonate, exposed bone in the maxillofacial region that has persisted for more than 8 weeks and no history of radiation therapy to the jaws is characteristic for MRONJ. Multiple staging systems have been proposed for MRONJ based on clinical and radiographic features as at risk, stage $0,1,2$ and 34 .

The medical management of MRONJ is aimed to control infection, minimize necrosis progression and promote tissue healing. ${ }^{9}$ Use of systemic antibiotics and oral antiseptic rinses such as Chlorhexidine gluconate are recommended. Use of both these strategies along Vitamin E led to the healing of the necrosed bone in our patient. Other treatment options available are hyperbaric oxygen therapy, low level laser therapy, ozone therapy and platelet rich plasma in postsurgical wound. ${ }^{10}$ Surgical treatments include sequestrectomy, debridement, resection, immediate reconstruction. Surgical treatment may also include extraction of teeth within exposed necrotic bone. In the case in question, sequestrectomy was done followed by medical management with antibiotics and antiseptic mouth rinse.

\section{CONCLUSIONS}

Osteonecrosis of jaw has constantly been researched on and has been renamed as BRONJ, ARONJ and MRONJ based on the medication responsible. Careful history taking to review medication in oncology patients with history of chemotherapy (antiresorptive drugs) and liaisoning with medical oncologist prior to any invasive dental treatment on these patients and treating with bisphosphonates / denosumab / antiangiogenic drugs is absolutely necessary to prevent the occurrence of consequences such as MRONJ. Role of oral medicine physicians in prevention and treating osteochemonecrosis is inevitable as early prevention and accurate treatment increase the quality of life in these groups of patients.

Financial or Other Competing Interests: None.

\section{REFERENCES}

[1] Drake MT, Clarke BL, Khosla S. Bisphosphonates: mechanism of action and role in clinical practice. Mayo Clin Proc 2008;83(9):1032-45.

[2] Marx RE. Pamidronate (Aredia) and zoledronate (Zometa) induced avascular necrosis of the jaws: a growing epidemic. J Oral Maxillofac Surg 2003;61(9):1115-7.

[3] Ruggiero SL, Dodson TB, Fantasia J, et al. American association of oral and maxillofacial surgeons position paper on medication-related osteonecrosis of the jaw2014 update. J Oral Maxillofac Surg 2014;72(10):193856.

[4] Rosella D, Papi P, Giardino R, et al. Medication-related osteonecrosis of the jaw: clinical and practical guidelines. J Int Soc Prev Community Dent 2016;6(2):97-104.

[5] Kajizono M, Sada H, Sugiura Y, et al. Incidence and risk factors of osteonecrosis of the jaw in advanced cancer patients after treatment with zoledronic acid or denosumab: a retrospective cohort study. Biol Pharm Bull 2015;38(12):1850-5.

[6] Vahtsevanos K, Kyrgidis A, Verrou E, et al. Longitudinal cohort study of risk factors in cancer patients of bisphosphonate-related osteonecrosis of the jaw. J Clin Oncol 2009;27(32):5356-62.

[7] Kalra S, Jain V. Dental complications and management of patients on bisphosphonate therapy: a review article. J Oral Biol Craniofac Res 2013;3(1):25-30.

[8] Ruggiero SL, Dodson TB, Assael LA, et al. American association of oral and maxillofacial surgeons position paper on bisphosphonate-related osteonecrosis of the jaws--2009 update. J Oral Maxillofac Surg 2009;67(5 Suppl):2-12.

[9] Beth-Tasdogan NH, Mayer B, Hussein $\mathrm{H}$, et al. Interventions for managing medication-related osteonecrosis of the jaw. Cochrane Database Syst Rev 2017;10(10):CD012432.

[10] Heggendorn FL, Leite TC, Cunha KSG, et al. Bisphosphonate-related osteonecrosis of the jaws: report of a case using conservative protocol. Spec Care Dentist 2016;36(1):43-7. 